Tôhoku Math. Journ.

$32(1980)$, 155-168.

\title{
STABILITY THEORY FOR COUNTABLY INFINITE SYSTEMS OF DIFFERENTIAL EQUATIONS
}

\author{
Dedicated to Professor Taro Yoshizawa on his sixtieth birthday \\ RichaRd K. MilleR* AND ANThony N. MiChel**
}

(Received June 12, 1978, revised October 9, 1978)

\begin{abstract}
New stability results for a class of countably infinite systems of differential equations are established. We consider those systems which may be viewed as an interconnection of countably infinitely many free or isolated subsystems. Throughout, the analysis is accomplished in terms of simpler subsystems and in terms of the system interconnecting structure. This approach makes it often possible to circumvent difficulties usually encountered in the application of the Lyapunov approach to complex systems with intricate structure. Both scalar Lyapunov functions and vector Lyapunov functions are used in the analysis. The applicability of the present results is demonstrated by means of several motivating examples, including a neural model.
\end{abstract}

1. Introduction. In the present paper we establish new stability results for a class of countably infinite systems of ordinary differential equations. We consider those systems which may be viewed as an interconnection of countably many free or isolated subsystems (which are described by ordinary differential equations defined on finite dimensional spaces). Such systems are often called interconnected systems, composite systems, decentralized systems, large scale systems, and the like (see Michel and Miller [5]). As in [5], our objective will be to analyze interconnected systems in terms of their simpler subsystems and in terms of their interconnecting structure. In this way it is frequently possible to circumvent difficulties usually encountered when the Lyapunov approarch is applied to complex systems with intricate structure.

For existing results dealing with stability and well posedness of countably infinite systems of differential equations, the reader is referred to Persidskii [7, 8], Leung et al. [4], Shaw [10, 11] and Deimling [2].

This paper consists of several parts. First we introduce initial value

* This research was supported by the National Science Foundation under grant ENG $77-28446$.

** This research was supported by the National Science Foundation under grant ENG 77-28446 and by the Engineering Research Institute, Iowa State University. 
problems which are characterized by countably infinite systems of equations, which are well posed, and which may be viewed as interconnected systems. Next, we establish several stability results which are obtained via scalar Lyapunov functions (consisting of a weighted sum of Lyapunov functions for the free subsystems). Next, we utilize infinite vector Lyapunov functions in our analysis (whose components are Lyapunov functions for the isolated subsystems). Throughout, several motivating examples are provided, including a neural model, to demonstrate the applicability of the method advanced.

2. Countably infinite systems of differential equations. A. Initial value problem. In this paper we are concerned with the initial value problem

$$
\dot{z}_{n}=h_{n}(t, x), \quad z_{n}(0)=c_{n}
$$

for $n=1,2,3, \cdots$. Here $x$ is the infinite dimensional column vector $x=\left(z_{1}^{T}, z_{2}^{T}, \cdots, z_{n}^{T}, \cdots\right)^{T} \in R^{w}, z_{n} \in R^{m_{n}}, D \subset R^{\omega}$ and $h_{n}: R^{+} \times D \rightarrow R^{m_{n}}$. The infinite product $R^{*}=R^{m_{1}} \times R^{m_{2}} \times \cdots \times R^{m_{n}} \times \cdots$ is given the usual product topology. Since this topology is equivalent to introducing the metric

$$
\rho(x, \bar{x})=\sum_{n=1}^{\infty}\left|z_{n}-\bar{z}_{n}\right|\left(1+\left|z_{n}-\bar{z}_{n}\right|\right)^{-1} 2^{-n}
$$

then $R^{*}$ is a convex Fréchet space (see, e.g., [9]).

A solution of (1) is a function $x:[0, b] \rightarrow D$ for some $b>0$ such that $z_{n} \in C_{n}^{1}[0, b], z_{n}(0)=c_{n}$ and $\dot{z}_{n}(t)=h_{n}(t, x(t))$ for all $t \in[0, b]$ and for all $n=1,2,3, \cdots$.

REMARK 1. An alternate way to view the above initial value problem is to fix a Banach space $X$ of real sequences and to think of (1)

$$
\dot{x}=h(t, x), \quad x(0)=c
$$

as a problem in $X$. In this case an $X$-solution is a function $x:[0, b] \rightarrow X$ which is continuously differentiable in the $X$-sense and satisfies ( $\left.1^{\prime}\right)$. Clearly, an $X$-solution for $\left(1^{\prime}\right)$ is also a solution in our sense (for (1)). However, in the present paper we shall not concern ourselves with Banach space settings for this initial value problem. Our approach has the advantage that well-posedness (i.e., existence, uniqueness and continuation results for (1)) poses no problems while it may pose serious difficulties in a Banach space setting (for $\left(1^{\prime}\right)$ ). The disadvantage of our approach is that before applying stability results, it is usually necessary to argue separately that the solutions $x(t)$ of (1) remain in a bounded 
set $D$.

B. Interconnected systems. Frequently we may regard system (1) as an interconnected system or a composite system of the form

$$
\dot{z}_{n}=f_{n}\left(t, z_{n}\right)+g_{n}(t, x), \quad z_{n}(0)=c_{n},
$$

$n=1,2,3, \cdots$, where in the notation of $(1), h_{n}(t, x) \stackrel{\Delta}{=} f_{n}\left(t, z_{n}\right)+g_{n}(t, x)$. We view $(\Sigma)$ as an interconnection of countably infinitely many isolated or free subsystems described by equations of the form

$$
\dot{w}_{n}=f_{n}\left(t, w_{n}\right),
$$

$n=1,2, \cdots$. The terms $g_{n}(t, x), n=1,2, \cdots$, comprise the interconnecting structure of system $(\Sigma)$.

C. Well-posedness. We shall assume that for some constants $r_{n}>0$ the set $D=D_{k}$ for some $k>0$ where $D_{k}=\left\{x=\left(z_{1}^{T}, z_{2}^{T}, \cdots\right)^{T} \in R^{\omega}:\left|z_{n}\right| \leqq\right.$ $k r_{n}$ for $\left.n=1,2,3, \cdots\right\}$. We shall also make the following additional assumptions.

(A-1): $f_{n}: R^{+} \times R^{m_{n}} \rightarrow R^{m_{n}}, f_{n}(t, 0)=0$ for all $t \geqq 0, f_{n}$ is locally Lipschitz continuous with Lipschitz constant independent of $t$ and for $\left|z_{n}\right| \leqq k r_{n}$, this Lipschitz constant is $L_{n 0}(k)$.

(A-2): $\quad g_{n}: R^{+} \times R^{\omega} \rightarrow R^{m_{n}}, \quad g_{n}(t, 0)=0$ for all $t \geqq 0$, and for any $k>0$ there exist constants $L_{n j}(k) \geqq 0$ such that $\sum_{j=1}^{\infty} L_{n j}(k) r_{j}<\infty$ and such that $\left|g_{n}(t, x)-g_{n}\left(t, x^{*}\right)\right| \leqq \sum_{j=1}^{\infty} L_{n j}(k)\left|z_{j}-z_{j}^{*}\right|$ for all $x, x^{*} \in D_{k}$, for all $t \geqq 0$, and all $n=1,2, \cdots$.

Theorem 1. (See Deimling [2, Corollary 6.2, p. 86]). If (A-1) and (A-2) are true for some $k$, if $\left|c_{n}\right| \leqq \alpha k r_{n}$ for some $\alpha \in(0,1)$ and for all $n \geqq 1$ and if

(A-3): there is an $M>0$ such that

$$
L_{n 0}(k) r_{n}+\sum_{j=1}^{\infty} L_{n j}(k) r_{j} \leqq M r_{n} \quad \text { for all } n \geqq 1,
$$

then $(\Sigma)$ has a unique solution $x(t, c)$ which will exist at least on the interval $0 \leqq t \leqq(1-\alpha) M^{-1}=T$ (where $c^{T}=\left(c_{1}^{T}, c_{2}^{T}, \cdots\right)$ and $\left.x(0, c)=c\right)$.

COROLlaRy 1. If in Theorem 1 the constants $L_{n j}$ can be chosen independent of $k$, then the solution will exist for all $t \geqq 0$ and on any interval $[0, T]$ there will exist $k$ such that $x(t) \in D_{k}$ for $0 \leqq t \leqq T$.

Proof. In this case $L_{n j}(k)=L_{n j}$ works for any $D_{k}$. Thus Equation (3) remains true when $r_{j}$ is replaced by $k r_{j}$ for any $k>0$. Given $\left\{c_{n}\right\}$, compute $x(t, c)$ on $[0, T], T=(1-\alpha) M^{-1}$. Since $\left|x_{n}(t)\right| \leqq k r_{n}$, we can 
continue the solution $x(t, c)=x(t-T, x(T, c))$ on $[T, 2 T]$ with $\left|x_{n}(2 T)\right| \leqq$ $k r_{n} / \alpha$. This process can be continued indefinitely to define $x(t, c)$ on $[m T,(m+1) T]$ with $\left|x_{n}(t)\right| \leqq k r_{n} \alpha^{-m}$ on the interval $m T \leqq t \leqq(m+1) T$. q.e.d.

We will also study finite approximations to $(\Sigma)$ which are of the form

$$
\left\{\begin{array}{l}
\dot{z}_{n}=f_{n}\left(t, z_{n}\right)+g_{n}\left(t,\left(z_{1}, z_{2}, \cdots, z_{N}, 0, \cdots\right)\right) \\
z_{n}(0)=c_{n}
\end{array}\right.
$$

$n=1,2, \cdots, N$, where $z_{n}(t) \equiv 0$ if $n>N$. In this case we let $x^{N}(t, c)$ denote the solution of $\left(\Sigma_{N}\right)$ and let $c^{N}=\left(c_{1}^{T}, c_{2}^{T}, \cdots, c_{N}^{T}, 0,0, \cdots\right)^{T}$.

Theorem 2. (See Deimling [2, Theorem 7.5, p. 101]). Under the hypotheses of Theorem 1 (or Corollary 1) we have

$$
\lim _{N \rightarrow \infty} x^{N}(t, c)=x(t, c)
$$

uniformly for $t$ in $[0, T]$ (or for $t$ on compact subsets of $R^{+}$).

3. Analysis by scalar Lyapunov functions. A. A stability result. Subsequently we will require the following assumptions.

(L-1): For isolated subsystem $\left(\mathscr{S}_{n}\right)$ there is a continuously differentiable function $v_{n}: R^{+} \times R^{m_{n}} \rightarrow R$ and three functions $\psi_{1 n}, \psi_{2 n}, \psi_{3 n} \in K$ and a constant $\sigma_{n} \in R$ such that

$$
\begin{aligned}
& \psi_{1 n}\left(\left|z_{n}\right|\right) \leqq v_{n}\left(t, z_{n}\right) \leqq \psi_{2 n}\left(\left|z_{n}\right|\right) \\
& D v_{n\left(S_{n}\right)}\left(t, z_{n}\right) \leqq \sigma_{n} \psi_{3 n}\left(\left|z_{n}\right|\right)
\end{aligned}
$$

for all $\left(t, z_{n}\right) \in R^{+} \times R^{m_{n}}$. Here we define $K$ as the class of all continuous functions with domain $R^{+}$and range in $R^{+}$having the properties that for any $\psi \in K, \psi(0)=0$ and $\psi\left(r_{1}\right)>\psi\left(r_{2}\right)$ whenever $r_{1}>r_{2}$. Also, $D v_{\left({ }_{n}\right)}$ denotes the derivative of $v_{n}$ along the solutions of $\left(\mathscr{S}_{n}\right)$.

For composite system $(\Sigma)$ we will utilize scalar Lyapunov functions of the form

$$
v(t, x)=\sum_{n=1}^{\infty} \lambda_{n} v_{n}\left(t, z_{n}\right)
$$

where the functions $v_{n}$ are defined in (L-1) and where the constants $\lambda_{n}>0$ will be chosen later to ensure that $v$ is defined and that along the solutions of composite system $(\Sigma)$, the derivative of $v$ with respect to $t$ is non-positive, i.e., $D v_{(},(t, x) \leqq 0$.

(L-2): Given $\psi_{3 n}$ in (L-1), there are constants $a_{n j} \in R$ such that $\nabla v_{n}\left(t, z_{n}\right)^{T} g_{n}\left(t,\left(z_{1}, \cdots, z_{N}, 0,0, \cdots\right)\right) \leqq \psi_{3 n}\left(\left|z_{n}\right|\right)^{1 / 2} \sum_{j=1}^{N} a_{n_{j}} \psi_{3 j}\left(\left|z_{j}\right|\right)^{1 / 2}$ for all $t \geqq 0$, 
$z_{n} \in R^{m_{n}}, n=1,2, \cdots, N$ and $N=1,2, \cdots$.

We will find it useful to define the matrix $R_{N}=\left[r_{i j}^{N}\right]$ and the matrix $S_{N}$ by

$$
r_{i j}^{N}= \begin{cases}\lambda_{i}\left(\sigma_{i}+a_{i i}\right) & \text { if } i=j \\ \lambda_{i} a_{i j} & \text { if } i \neq j\end{cases}
$$

and

$$
S_{N}=\left(R_{N}+R_{N}^{T}\right) / 2 .
$$

We shall call the trivial solution $x \equiv 0$ of the system $(\Sigma)$ stable with respect to a set $D$ if for any $\varepsilon>0$ there is a $\delta>0$ such that when $c \in D$ and $\rho(c, 0)<\delta$ then $\rho(x(t, c), 0)<\varepsilon$ for all $t \geqq 0$. (Equivalently one can say that for any $\varepsilon>0$ and any integer $J_{0} \geqq 1$ there exists $\delta>0$ and an integer $J_{1} \geqq 1$ such that when $e \in D$ and $\left|c_{n}\right| \leqq \delta$ for $1 \leqq n \leqq J_{1}$ then $\left|z_{n}(t, c)\right|<\varepsilon$ for all $t \geqq 0$ and for $n=1,2, \cdots, J_{0}$.) We call the trivial solution $x \equiv 0$ asymptotically stable with respect to $D$ if it is stable with respect to $D$ and if for any initial condition $c \in D$ the solution $x(t, c) \rightarrow 0$ as $t \rightarrow \infty$.

LeMmA 1. Suppose that (A-1), (A-2), (A-3), (L-1) and (L-2) are true and assume that there exist constants $\lambda_{n}>0$ such that $\sum_{n=1}^{\infty} \lambda_{n} \psi_{2 n}\left(k r_{n}\right)<\infty$. If matrix $S_{N}$ is negative semi-definite for all $N$ sufficiently large, then $v(t, x(t, c))$ is defined, continuous and nonincreasing in $t \in\left[0,(1-\alpha) M^{-1}\right]$ for any initial condition $c \in D_{\alpha l}$.

Proof. Suppose that $x(t, c)$ exists on $[0, b]$. By Theorem $2 x^{N}(t, c) \rightarrow$ $x(t, c)$ as $N \rightarrow \infty$ uniformly on $[0, b]$. Thus, $\lim _{N \rightarrow \infty} v\left(t, x^{N}(t, c)\right)=v(t, x(t, c))$ uniformly on $[0, b]$. Moreover, $(d / d t) v\left(t, x^{N}(t, c)\right)=\sum_{n=1}^{N} \lambda_{n} D v_{n\left(\sigma_{n}\right)}\left(t, z_{n}\right)+$ $\lambda_{n} \nabla v_{n}\left(t, z_{n}\right)^{T} g_{n}\left(t, x^{N}(t, c)\right) \leqq \sum_{n=1}^{N} \lambda_{n}\left[\sigma_{n} \psi_{3 n}\left(\left|z_{n}\right|\right)+\sum_{j=1}^{N} \lambda_{n} a_{n j} \psi_{3 n}\left(\left|z_{n}\right|\right)^{1 / 2} \psi_{3 j}\left(\left|z_{j}\right|\right)^{1 / 2}\right]=$ $\psi_{N}^{T} R_{N} \psi_{N}=\psi_{N}^{T}\left[\left(R_{N}+R_{N}^{T}\right) / 2\right] \psi_{N}=\psi_{N}^{T} S_{N} \psi_{N} \leqq 0$ where $\psi_{n}=\left(\psi_{1}\left(\left|z_{1}\right|\right)^{1 / 2}, \psi_{2}\left(\left|z_{2}\right|\right)^{1 / 2}\right.$, $\left.\cdots, \psi_{N}\left(\left|z_{N}\right|\right)^{1 / 2}\right)$. Since $v\left(t, x^{N}(t, c)\right)$ is nonincreasing in $t$ (for all $N$ sufficiently large), then so is the limit $v(t, x(t, c))$.

q.e.d.

THEOREM 3. Suppose that (A-1), (A-2), and (A-3) are true with Lipschitz constants $L_{n j}$ independent of $k$ and suppose that (L-1) and (L-2) are true. Assume that the constants $\lambda_{n}$ can be picked so that the matrix $S_{N}$ is negative semi-definite (see (5) and (6)) for all $N$ sufficiently large and so that $\sum_{n=1}^{\infty} \lambda_{n} \psi_{2 n}\left(r_{n}\right)<\infty$. Define $D=D_{k}$ for $k=1$. Then the trivial solution $x=0$ of composite system $(\Sigma)$ is stable with respect to the set $D$.

Proof. Fix $\varepsilon$ and $J_{0}$. Since $\psi_{1 n} \in K$ for all $n \geqq 1$ and since $v(t, x) \geqq$ $\sum_{n=1}^{\infty} \lambda_{n} \psi_{1 n}\left(\left|z_{n}\right|\right) \geqq \sum_{n=1}^{J_{0}} \lambda_{n} \psi_{1 n}\left(\left|z_{n}\right|\right) \geqq \max \left\{\lambda_{n} \psi_{1 n}\left(\left|z_{n}\right|\right): 1 \leqq n \leqq J_{0}\right\}$, then there 
exists $m>0$ such that $v(t, x) \geqq m$ if for some $n$ between 1 and $J_{0}$ we have $\left|z_{n}\right| \geqq \varepsilon$. Pick $J_{1}$ so large that $\sum_{n=J_{1}+1}^{\infty} \lambda_{n} \psi_{2 n}\left(r_{n}\right)<m / 2$ and pick $\delta>0$ so small that $\sum_{n=1}^{J_{1}} \lambda_{n} \psi_{2 n}(\delta)<m / 2$. If $\left|c_{n}\right| \leqq \delta$ for $1 \leqq n \leqq J_{1}$, then by Lemma $1 v(t, x(t, c)) \leqq v(0, c)<m$. Thus $\left|z_{n}(t, c)\right|<\varepsilon$ for all $t \geqq 0$ and for $1 \leqq n \leqq J_{0}$.

q.e.d.

EXAMPLE 1. Consider the countable system of scalar equations

$$
\left\{\begin{array}{l}
\dot{z}_{1}=-z_{1} \\
\dot{z}_{n}=-z_{n}+z_{n-1}, \quad n \geqq 2 .
\end{array}\right.
$$

We can view (7) as an interconnection of countably infinite many free subsystems described by

$$
\dot{z}_{n}=-z_{n},
$$

$n=1,2, \cdots$, with interconnecting structure specified by $g_{n}(t, x)=z_{n-1}$, $n \geqq 2$.

For any $r>0$ we can set $r_{n}=r$ for all $n \geqq 1$ and see that (A-1) and (A-2) are satisfied for (7).

For $\left(\mathscr{P}_{n}\right)$ we choose $v_{n}\left(z_{n}\right)=z_{n}^{2} / 2$. In the notation of (L-1) and (L-2) we have $\psi_{1 n}\left(\left|z_{n}\right|\right)=\psi_{2 n}\left(\left|z_{n}\right|\right)=z_{n}^{2} / 2, \psi_{3 n}\left(\left|z_{n}\right|\right)=z_{n}^{2}, \sigma_{n}=-1, a_{n, n-1}=1$ for $n \geqq 2$, and $a_{i j}=0$ for all other $i$ and $j$. Thus, (L-1) and (L-2) are satisfied as well.

To show that the remaining hypotheses of Theorem 3 are satisfied, we let

$$
A=\left[\begin{array}{ccccc}
1 & 0 & \cdots & 0 & 0 \\
-1 & 1 & \cdots & 0 & 0 \\
\cdots & \cdots & \cdots & \\
0 & 0 & \cdots & -1 & 1
\end{array}\right]
$$

If $0<a_{1}<a_{2}<\cdots<a_{N}$ and $p=\left(a_{1}, a_{2}, \cdots, a_{N}\right)^{T}$, then $A p>0$ (i.e., each component of vector $A p$ is positive). If $b_{1}>b_{2}>\cdots>b_{N}>0$ and $q=$ $\left(b_{1}, b_{2}, \cdots, b_{N}\right)^{T}$ then $A^{T} q>0$. Define $e_{j}=b_{j} / a_{j}, 1 \leqq j \leqq N, \quad W=$ $\operatorname{diag}\left[e_{1}, e_{2}, \cdots, e_{N}\right]$, and $B=\left[b_{i j}\right]=\left(W A+A^{T} W\right)$. Then $b_{i j} \leqq 0$ for all $i \neq j$ and $B p=\left(W A+A^{T} W\right) p=W(A p)+A^{T} q>0$. It follows that $B$ is an $M$-matrix [3,6] (i.e., $b_{i j} \leqq 0$ for all $i \neq j$ and all successive principal minors of $B$ are positive) and $B=B^{T}$ is positive definite.

Using the notation of Equations (4)-(6) we choose $\lambda_{j}=e_{j}$. Then the matrix $B$ constructed above equals the matrix $-2 S_{N}$. Thus $S_{N}$ is negative definite. The only remaining question is whether the resulting $\lambda_{n}$ are summable. However, this is easy to arrange. For example, if $a_{n}=$ $2^{n / 2}, b_{n}=2^{-n / 2}$, then $\lambda_{n}=2^{-n}$ will do. 
All hypotheses of Theorem 3 are now satisfied and the trivial solution $x=0$ of system (7) is stable with respect to $D_{k}$ for any $k>0$.

The system of equations $(\Sigma)$ can in this particular case be solved explicitly to obtain $z_{n}(t)=e^{-t}\left[c_{n}+c_{n-1} t+c_{n-2} t^{2} / 2 !+\cdots+c_{1} t^{n-1} /(n-1)\right.$ ! $]$. Thus, in the present example all solutions tend to zero as $t \rightarrow \infty$. We develop below some theory to predict this asymptotic stability.

B. An invariance result. The purpose of the next example is to provide motivation for the next result.

EXAmPle 2. Consider the system of scalar equations

$$
\dot{z}_{n}=-z_{n}+2 \sum_{m=n+1}^{\infty}\left(g_{n m} / \lambda_{n}\right) z_{m},
$$

$n=1,2, \cdots$, where $g_{n m} \geqq 0, g_{n, n+1}>0$, and the $g$ are assumed to be such that $\sum_{n=1}^{\infty} \sum_{m=n+1}^{\infty} g_{n m}<\infty$ and where $\lambda_{n}=\left(\sum_{m=1}^{n-1} g_{m n}+\sum_{m=n+1}^{\infty} g_{n m}\right)>0$. Reversing the order of summation in the first sum below we see that $\sum_{n=1}^{\infty} \lambda_{n}=\sum_{n=1}^{\infty}\left(\sum_{m=1}^{n-1} g_{m n}+\sum_{m=n+1}^{\infty} g_{n m}\right)=2 \sum_{n=1}^{\infty} \sum_{m=n+1}^{\infty} g_{n m}<\infty$.

For each isolated subsystem $\left(\mathscr{S}_{n}\right)$ of $(\Sigma)$, given by

$$
\dot{z}_{n}=-z_{n}
$$

we choose $v_{n}\left(z_{n}\right)=z_{n}^{2} / 2$ and for composite system (9) we choose the Lyapunov function $v(x)=\sum_{n=1}^{\infty} \lambda_{n} v_{n}\left(z_{n}\right)$. Since the system of equations (9) is upper triangular, rather than using finite approximations, we can equivalently compute directly that $-D v_{(9)}(x)=\sum_{n=1}^{\infty}\left[\left(\sum_{m=1}^{n-1} g_{m n}+\right.\right.$ $\left.\left.\sum_{m=n+1}^{\infty} g_{n m}\right) z_{n}^{2}-2 \sum_{m=n+1}^{\infty} g_{n m} z_{n} z_{m}\right]=\sum_{m=1}^{\infty} \sum_{n=m+1}^{\infty} g_{m n} z_{n}^{2}+\sum_{n=1}^{\infty} \sum_{m=n+1}^{\infty} g_{n m}\left(z_{n}^{2}-\right.$ $\left.2 z_{n} z_{m}\right)=\sum_{n=1}^{\infty} \sum_{m=n+1}^{\infty} g_{n m}\left(z_{n}-z_{m}\right)^{2}$. Thus, $D v_{(9)}(x) \leqq 0$ and with $r_{n}=r$ for all $n \geqq 1$ we obtain stability for system (9) by Theorem 3 .

REMARK 2. In the above example it is natural to ask if more can be asserted. Specifically, do the solutions (of (9) in Example 2) tend to the set $E=\left\{\left(z_{1}, z_{2}, \cdots, z_{n}, \cdots\right): z_{1}=z_{2}=\cdots=z_{n}=\cdots\right\}$ ? This is a natural question, since above $D v_{(9)}(x)$ is negative definite with respect to the set $E$. Is it possible to prove an invariance theorem (see [13] or [1, Section 5.4]) for this system (i.e., do the solutions tend to an invariant subset of $E$ ?)? The next result provides some answers along such lines.

THEOREM 4. Assume that the hypotheses of Theorem 3 are true. Assume there exists $k>0$ such that $x(t, c) \in D_{k}$ for all $t \geqq 0$ and for all $c \in D$. Suppose that $D v_{(\Sigma)}(t, x) \leqq-W(x)$ where $W(x)$ is positive definite with respect to a set $E \subset D_{k}$ (i.e., for every $\varepsilon>0$ there is a $\delta>0$ such that if $x \in D_{k}$ and $\rho(x, E) \geqq \varepsilon$ then $\left.W(x) \geqq \delta\right)$. If the functions $f_{n}, g_{n}$ and $v_{n}$ are all independent of $t$, then for any $c \in D$ 
(i ) $x(t, c)$ tends to $E$ as $t \rightarrow \infty$, and

(ii) the positive limit set $\Omega(x(\cdot, c))$ is an invariant set with respect to $(\Sigma)$.

Proof. Assume that (i) is not true. Then there is a monotone sequence $\left\{t_{n}\right\}$ such that $t_{n} \rightarrow \infty$ and an $\varepsilon>0$ such that $\rho\left(x\left(t_{n}, c\right), E\right) \geqq 2 \varepsilon$. Since $D_{k}$ is a compact set and $h_{n}=f_{n}+g_{n}$ is continuous there, then $h_{n}$ is bounded on $D_{k}$. Thus $z_{n}(t)$ is uniformly continuous on $0 \leqq t<\infty$ for each $n \geqq 1$, i.e., there is an $\eta>0$ such that $\rho(x(t, c), E) \geqq \varepsilon$ for $\left|t-t_{n}\right| \leqq \eta$. Also, there is a $\delta>0$ such that $W(x) \geqq \delta$ if $\rho(x, E) \geqq \varepsilon$. Thus for $t \geqq$ $t_{N}+\eta$ we have

$$
0 \leqq v(x(t)) \leqq v(c)-\int_{0}^{t} W(x(s)) d s \leqq v(c)-\sum_{j=1}^{N} \int_{t_{n}-\eta}^{t_{n}+\eta} \delta d s .
$$

The last expression is negative when $N>v(c) /(2 \delta \eta)$. This contradiction completes the proof of (i).

To prove (ii) note that each component $z_{n}(t)$ is uniformly continuous and bounded on $0 \leqq t<\infty$. Let $d \in \Omega(x(\cdot, c))$ and let $t_{n} \rightarrow \infty$ be a sequence such that $x\left(t_{n}, c\right) \rightarrow d=\left(d_{1}, d_{2}, \cdots\right)$. Then $z_{m}\left(t+t_{m}, c\right)$ is an equicontinuous, uniformly bounded sequence of functions defined on $-T \leqq t \leqq T$ for any $T>0$. By possibly taking a subsequence we can assume that $\lim _{n \rightarrow \infty} z_{m}\left(t+t_{n}, c\right)=y_{m}(t)$ exists uniformly for $t$ on compact subsets of $-\infty<t<\infty$. Thus, as $n \rightarrow \infty$ we get $z_{m}\left(t+t_{n}, c\right)=z_{m}\left(t_{n}, c\right)+$ $\int_{0}^{t} h_{m}\left(x\left(s+t_{n}, c\right)\right) d s$. In the limit this is $y_{m}(t)=d_{m}+\int_{0}^{t} h_{m}(y(s)) d s$. This means that $y(t)=x(t, d)$ for $-\infty<t<\infty$.

q.e.d.

REMARK 3. Part (i) of Theorem 4 covers Example 1 above. Indeed in that example it is easy to compute that $D v_{(7)}(t, x) \leqq(-3 / 8) z_{1}^{2}-$ $(1 / 4) \sum_{n=2}^{\infty} z_{n}^{2} / 2^{n} \leqq(-1 / 8) v(t, x)$ if we choose $\lambda_{n}=2^{-n}$. Since $v(t, x)=v(x)$ is positive definite, then we can take $E=$ the origin and assert asymptotic stability of (7) with respect to $D_{k}$ for any $k>1$.

REMARK 4. In Example 2 we see that given any solution $x(t, c)$ with $\left|z_{n}(t, c)\right| \leqq k$ for some $k$ and for all $t \geqq 0$, then by Theorem $4, x(t, c) \rightarrow$ $E=\left\{\left(z_{1}, z_{2}, \cdots\right)^{T}: z_{1}=z_{2}=\cdots\right\}$. Indeed $x(t, c)$ tends to the largest invariant subset of $E$, which we denote by $M$. On $M$ the system of equations (9) reduces to $\dot{z}_{n}=\left(-1+\left(2 / \lambda_{n}\right) \sum_{m=n+1}^{\infty} g_{n m}\right) z_{n}=\eta z_{n}, n=1,2,3, \cdots$, where $\eta=-1+\left(2 / \lambda_{n}\right) \sum_{m=n+1}^{\infty} g_{n m}$ must be independent of $n$. Indeed, since the components $z_{n}$ are bounded on $-\infty<t<\infty$, either $\eta=0$ or $z_{n} \equiv 0$. Now $\eta=-1+\left(2 / \lambda_{n}\right) \sum_{m=n+1}^{\infty} g_{n m}=\left(\sum_{m=n+1}^{\infty} g_{n m}-\sum_{m=1}^{n-1} g_{n m}\right) /\left(\sum_{m=n+1}^{\infty} g_{n m}+\sum_{m=1}^{n-1} g_{m n}\right)$. Therefore either $M$ is just the origin or else $\sum_{m=n+1}^{\infty} g_{n m}=\sum_{m=1}^{n-1} g_{m n}$ $(n=1,2,3, \cdots)$. This is impossible since for $n=1, \sum_{m=1}^{0} g_{m n}$ means zero 
while $\sum_{m=2}^{\infty} g_{1 m}=\lambda_{1}>0$.

In Theorem 4 the hypothesis that $x(t, c) \in D_{k}$ for all $t \geqq 0$ for some $k>0$ is needed only to ensure that $x(t, c)$ is uniformly continuous on $[0, \infty)$. This can also be assured in other ways, e.g., Theorem 5 . We say that system $(\Sigma)$ is row-finite if for each $n \geqq 1$ there is an integer $m(n) \geqq 1$ such that $h_{n}(t, x)=h_{n}\left(t, x_{1}, \cdots, x_{m(n)}\right)$ depends only on $t$ and $x_{j}$ for $1 \leqq j \leqq m(n)$. The proof of the following result is similar to that of Theorem 4 above.

THEOREM 5. Assume that the hypotheses of Theorem 3 are true. Suppose that $D v_{(\Sigma)}(t, x) \leqq-W(x)$ where $W(x)$ is positive definite with respect to a set $E$. If $f_{n}, g_{n}$ and $v_{n}$ are all independent of $t$ and $(\Sigma)$ is row finite, then for any $c \in D$ conclusions (i) and (ii) of Theorem 4 remain true.

REMARK 5. In Example 2 if for each $n$ there exists $m(n)$ such that $g_{n m}=0$ when $m \geqq m(n)$ and if $\sup _{n}\left|c_{n}\right|<\infty$, then $x(t, c) \rightarrow 0$ as $t \rightarrow \infty$.

EXAMPLE 3. As in Example 2 suppose that $g_{n m} \geqq 0$ with $g_{n, n+1}>0$ and $\sum_{n=1}^{\infty} \sum_{m=n+1}^{\infty} g_{n m}<\infty, \quad \lambda_{n} \stackrel{\Delta}{=} \sum_{m=1}^{n-1} g_{m n}+\sum_{m=n+1}^{\infty} g_{n m}>0$ and consider the system of equations

$$
\begin{aligned}
& \dot{z}_{1}=-z_{1}+\sum_{m=2}^{\infty}\left(g_{1 n} / \lambda_{1}\right) z_{m} \\
& \dot{z}_{n}=-z_{n}+\sum_{m=n+1}^{\infty}\left(g_{n m} / \lambda_{n}\right) z_{m}+\sum_{m=1}^{n-1}\left(g_{m n} / \lambda_{n}\right) z_{m}, \quad n \geqq 2 .
\end{aligned}
$$

If $v(x)=\sum_{n=1}^{\infty} \lambda_{n} z_{n}^{2} / 2$, then by a calculation much like that in the last example we obtain $-D v_{(\Sigma)}(x)=\sum_{n=1}^{\infty}\left[\lambda_{n} z_{n}^{2}-\sum_{m=n+1}^{\infty} g_{n m} z_{n} z_{m}-\sum_{m=1}^{n-1} g_{m n} z_{n} z_{m}\right]=$ $\sum_{n=1}^{\infty}\left[\sum_{m=n+1}^{\infty} g_{n m} z_{n}^{2}-\sum_{m=n+1}^{\infty} g_{n m} z_{n} z_{m}\right]+\sum_{n=1}^{\infty} \sum_{m=1}^{n-1} g_{m n} z_{n}^{2}-\sum_{n=1}^{\infty} \sum_{m=1}^{n-1} g_{m n} z_{n} z_{m}=$ $\sum_{n=1}^{\infty} \sum_{m=n+1}^{\infty} g_{n m}\left(z_{n}^{2}-z_{n} z_{m}\right)+\sum_{m=1}^{\infty} \sum_{n=m+1}^{\infty} g_{m n}\left(z_{n}^{2}-z_{n} z_{m}\right)=\sum_{n=1}^{\infty} \sum_{m=n+1}^{\infty} g_{n m}\left(z_{n}^{2}-\right.$ $\left.z_{n} z_{m}+z_{m}^{2}-z_{m} z_{n}\right)=\sum_{n=1}^{\infty} \sum_{m=n+1}^{\infty} g_{n m}\left(z_{n}-z_{m}\right)^{2}$. If $\sup _{n}\left|c_{n}\right|<\infty$, then the solution $x(t, c) \rightarrow E$ as $t \rightarrow \infty$ where $E=\left\{\left(z_{1}, z_{2}, z_{3}, \cdots\right): z_{1}=z_{2}=z_{3}=\cdots\right\}$, provided either $\sup _{t \geqq 0, n \geq 1}\left|z_{n}(t, c)\right|<\infty$ or the system is row-finite. An invariant subset of $E$ has solutions with the property $z_{1} \equiv z_{2} \equiv z_{3} \equiv \cdots$ so that $\dot{z}_{n}=-z_{n}+\sum_{m=n+1}^{\infty}\left(g_{n m} / \lambda_{n}\right) z_{n}+\sum_{m=1}^{n-1}\left(g_{m n} / \lambda_{n}\right) z_{n}=-\left(1 / \lambda_{n}\right)\left(\lambda_{n}-\sum_{m=n+1}^{\infty} g_{n m}-\right.$ $\left.\sum_{m=1}^{n-1} g_{m_{n}}\right) z_{n}$. By the definition of $\lambda_{n}$, these coefficients are all zero. Thus all points of the form $x^{T}=\left(z_{1}, z_{2}, \cdots\right)$ with $z_{1}=z_{2}=z_{3}=\cdots=K$ constant are solutions. (The origin is not an attractor.)

EXAMPLe 4. Consider the system of equations

$$
\left\{\begin{array}{l}
\dot{z}_{1}=-2 z_{1}+z_{2} \\
\dot{z}_{n}=z_{n-1}-2 z_{n}+z_{n+1}, \quad n \geqq 2 .
\end{array}\right.
$$


Let $\sum_{n=1}^{\infty} r_{n}^{2}<\infty, \quad \sup _{n}\left(r_{n+1}+r_{n-1}\right) / r_{n}=S<\infty$ and choose $v(x)=$ $(1 / 2) \sum_{n=1}^{\infty} z_{n}^{2}$. Then (A-3) is true with $M=S+2$ and $D v_{(11)}(x)=-z_{1}^{2}-$ $\sum_{n=1}^{\infty}\left(z_{n}-z_{n+1}\right)^{2} \leqq 0$. The solutions are uniformly bounded, $\sup _{n, t}\left|z_{n}(t, c)\right|<\infty$ and $x(t, c) \rightarrow 0$ as $t \rightarrow \infty$ by invariance.

C. Weak-coupling conditions. In our next result we consider some alternate hypotheses.

(L-3): For each free subsystem $\left(\mathscr{S}_{n}\right)$ there is a function $v_{n}: R^{+} \times$ $R^{m_{n}} \rightarrow R^{+}$, a constant $\sigma_{n} \in R$, a constant $L_{n} \geqq 0$, and functions $\psi_{j_{n}} \in K$, $j=1,2,3$, such that

$$
\begin{aligned}
& \psi_{1 n}\left(\left|z_{n}\right|\right) \leqq v_{n}\left(t, z_{n}\right) \leqq \psi_{2 n}\left(\left|z_{n}\right|\right) \\
& D v_{n\left(\mathscr{S}_{n}\right)}\left(t, z_{n}\right) \leqq \sigma_{n} \psi_{\beta_{3 n}}\left(\left|z_{n}\right|\right) \\
& \left|v_{n}\left(t, z_{n}\right)-v_{n}\left(t, z_{n}^{*}\right)\right| \leqq L_{n}\left|z_{n}-z_{n}^{*}\right|
\end{aligned}
$$

for all $t \geqq 0$ and for all $z_{n}, z_{n}^{*} \in R^{m_{n}}$.

(L-4): There are constants $a_{n m} \geqq 0$ such that $\left|g_{n}(t, x)\right| \leqq \sum_{m=1}^{N} a_{n m} \psi_{3 m}\left(\left|z_{m}\right|\right)$ whenever $x^{T}=\left(z_{1}^{T}, z_{*}^{T}, \cdots, z_{N}^{T}, 0,0, \cdots\right)$ for any $N \geqq 1$.

Also, in our next result we will employ Lyapunov functions for $(\Sigma)$ which are of the form $v(t, x)=\sum_{n=1}^{\infty} \lambda_{n} v_{n}\left(t, z_{n}\right)$ with constants $\lambda_{n}>0$ which we will further specify later. We will also utilize $N \times N$ matrices $R_{N}=\left[r_{n m}\right]$ specified by

$$
r_{n m}=\left\{\begin{array}{lll}
-\left(\sigma_{n}+L_{n} a_{n n}\right) & \text { if } & n=m \\
-L_{n} a_{n m} & \text { if } & n \neq m
\end{array} .\right.
$$

Finally, we will also require the following additional hypothesis:

(L-5): For each $N$ sufficiently large, $\Lambda_{N} R_{N} \geqq 0$, i.e., $\sum_{j=1}^{N} \lambda_{j} r_{j n} \geqq 0$ for $1 \leqq n \leqq N$.

Under the above assumptions we can easily compute $(d / d t) v\left(t, x^{N}(t, c)\right) \leqq$ $\sum_{n=1}^{N} \lambda_{n}\left\{\sigma_{n} \psi_{3 n}\left(\left|z_{n}\right|\right)+\sum_{m=1}^{N} L_{n} a_{n m} \psi_{3 m}\left(\left|z_{m}\right|\right)\right\}=-\Lambda_{N} R_{N} \psi_{N}(|x|)$ where $\Lambda_{N}=\left(\lambda_{1}, \lambda_{2}\right.$, $\left.\cdots, \lambda_{N}\right)$ and $\psi_{N}(|x|) \stackrel{\Delta}{=}\left(\psi_{31}\left(\left|z_{1}\right|\right), \cdots, \psi_{3 N}\left(\left|z_{N}\right|\right)\right)^{T}$. By assumption (L-5) it now follows that $\Lambda_{N} R_{N} \geqq 0$. Thus, $D v_{(\Sigma)}(t, x) \leqq 0$. Indeed, we have $D v_{(\Sigma)}(t, x) \leqq$ $-\sum_{n=1}^{\infty}\left(\sum_{j=1}^{\infty} \lambda_{j} r_{j n}\right) \psi_{3 n}\left(\left|z_{n}\right|\right)=-W(x)$. Moreover, $W(x)$ is positive definite with respect to the set $E^{*}=\left\{x=\left(z_{1}, z_{2}, z_{3}, \cdots\right): z_{n}=0\right.$ when $\left.\sum_{j=1}^{\infty} \lambda_{j} r_{j n}>0\right\}$.

Summarizing, we now have

THEOREM 6. If assumptions (A-1), (A-2) and (A-3) are true with Lipschitz constant $L_{n j}$ independent of $k>0$, if assumptions (L-4) and (L-5) are true, and if $\sum_{n=1}^{\infty} \lambda_{n} \psi_{2 n}\left(r_{n}\right)<\infty$, then

(i) the trivial solution $x=0$ of system $(\Sigma)$ is stable with respect to $D_{k}$ for $k=1$.

(ii) Let $M$ be the largest invariant subset of $E^{*}$. If $c \in D$ and 
either $x(t, c) \in D_{K}$ for all $t \geqq 0$ and some fixed $k>1$ or $(\Sigma)$ is row finite, then $x(t, c) \rightarrow M$ as $t \rightarrow \infty$.

EXAMPLE 5. We reconsider Example 4,

$$
\left\{\begin{array}{l}
\dot{z}_{1}=-2 z_{1}+z_{2} \\
\dot{z}_{n}=z_{n-1}-2 z_{n}+z_{n+1} \quad(n \geqq 2) .
\end{array}\right.
$$

For each isolated subsystem (13),

$$
\dot{z}_{n}=-2 z_{n},
$$

$n \geqq 1$, we choose Lyapunov functions of the form $v_{n}\left(z_{n}\right)=\left|z_{n}\right|$. Then $\psi_{1 n}\left(\left|z_{n}\right|\right)=\psi_{2 n}\left(\left|z_{n}\right|\right)=\left|z_{n}\right|, \quad \psi_{3 n}\left(\left|z_{n}\right|\right)=\left|z_{n}\right|, \quad \sigma_{n}=-2$ and $L_{n}=1$. Thus, (L-3) is satisfied. Hypothesis (L-4) is also satisfied with $a_{n, n-1}=a_{n, n+1}=1$ for $n \geqq 2, a_{12}=1$, and $a_{i j}=0$ for all other indices. Choose $c$ such that $\sum_{n=1}^{\infty}\left|c_{n}\right|<\infty$ and let $r_{n} \geqq\left|c_{n}\right|$ be such that $\sup _{n}\left(r_{n+1}+r_{n-1}\right) / r_{n}=S<\infty$ and $\sum_{n=1}^{\infty} r_{n}<\infty$ (e.g., $r_{n}=K / 2^{n}$ ). For system (12) we choose $v(x)=$ $\sum_{n=1}^{\infty} v_{n}\left(z_{n}\right)=\sum_{n=1}^{\infty}\left|z_{n}\right|$. Then $D v_{(12)}(x)=-2\left|x_{1}\right|+\left|x_{2}\right|+\sum_{n=2}^{\infty}\left(\left|x_{n-1}\right|-2\left|x_{n}\right|+\right.$ $\left.\left|x_{n+1}\right|\right)=-\left|x_{1}\right| \leqq 0$. Thus $\sup _{n} \sup _{t \geq 0}\left|z_{n}(t, c)\right|<\infty$. Moreover, $E^{*}=$ $\left\{x: z_{1}=0\right\}$ and if $x(t, c) \in M$, then $z_{1}(t, c)=0$ implies that $\dot{z}_{1}+2 z_{1}=z_{2}=0$. Similarly, $\dot{z}_{2}+2 z_{2}=z_{3}=0$, and so forth. Thus $M=\{(0,0, \cdots, 0, \cdots)\}$.

All the hypotheses of Theorem 6 are satisfied. The trivial solution of (12) is asymptotically stable with respect to $D_{k}$ for any $k>1$.

4. Analysis by vector Lyapunov functions. An (infinite) vector Lyapunov function approach can also be used to analyze certain stability problems for system $(\Sigma)$. For example, if $D v_{n\left(S_{n}\right)} \leqq \sigma_{n} v_{n}$ and if $\left|g_{n}(t, x)\right| \leqq$ $\sum_{m=1}^{\infty} a_{n m} v_{m}\left(t, z_{m}\right)$ for some sequence $a_{n m} \geqq 0$, then we can consider for system $(\Sigma)$ the infinite vector Lyapunov function

$$
v(t, x)=\left(v_{1}\left(t, z_{1}\right), v_{2}\left(t, z_{2}\right), \cdots\right)^{T} .
$$

This vector satisfies for $(\Sigma)$ the differential inequality $D v_{n} \leqq \sigma_{n} v_{n}+$ $L_{n} \sum_{m=1}^{\infty} a_{n m} v_{n}, \quad n=1,2, \cdots$ By the comparison results in [12] and Theorem 2 above we have $v_{n}\left(t, z_{n}(t, c)\right) \leqq w_{n}(t)$ for all $n \geqq 1$ and all $t \geqq 0$, where $w_{n}, n=1,2, \cdots$, are the solutions of the linear equations

$$
\dot{w}_{n}=\sigma_{n} w_{n}+\sum_{m=1}^{\infty} L_{n} a_{n m} w_{m} \quad(n \geqq 1)
$$

with $w_{n}(0) \geqq v_{n}\left(0, z_{n}(0, c)\right)$. Thus, if the stability properties of system $(\sigma)$ can be determined, then the stability properties of system $(\Sigma)$ can be inferred.

In our next example we consider a neural model which has been studied previously (see [4]). 
ExAmple 6. Consider the neural model given by the scalar equations

$$
\left\{\begin{array}{l}
\dot{z}_{n}=-\alpha_{n} z_{n}+f_{n}(x) \\
z_{n}(0)=c_{n}
\end{array}\right.
$$

where $\alpha_{n}>0, \quad 0<c_{n}<1, \quad f_{n}(x)=\left[1+\exp \left(-\gamma_{n}-\sum_{m=1}^{\infty} \beta_{n m} z_{m}\right)\right]^{-1}$, and $\sum_{m=1}^{\infty}\left|\beta_{n m}\right| \leqq b<\infty$ for each $n \geqq 1$. We will accomplish our analysis of (14) in several steps. In doing so we shall make the assumptions that $\beta_{n m} \geqq 0, \quad \alpha_{n}\left[1+\exp \left(-\gamma_{n}-\sum_{m=1}^{\infty} \beta_{n m}\right)\right]>1$, and there exists $\lambda_{n} \geqq 0$ such that $\sum_{n=1}^{\infty} \lambda_{n}<\infty$ and $\sum_{m=1}^{\infty} \lambda_{m} \alpha_{m} \beta_{m n}<\lambda_{m} \alpha_{m}(n \geqq 1)$.

(a) Claim. There is a critical point $x^{*}=\left(z_{1}^{*}, z_{2}^{*}, \cdots\right)^{T}$ with $0<$ $z_{n}^{*}<1$.

To verify this we define $S$ by $S=\left\{x=\left(z_{1}, z_{2}, \cdots\right)^{T}: 0 \leqq z_{n}<1\right\}$ and we define $T x$ by $T_{n} x=\alpha_{n}^{-1} f_{n}(x)$. Let $z^{0}=(0, \cdots, 0, \cdots)^{T}$ and $z^{n+1}=T\left(z^{n}\right)$. By monoticity, $z^{0} \leqq z^{1} \leqq z^{2} \leqq \cdots<(1,1, \cdots, 1, \cdots)=e$. This and continuity imply that $z^{m} \nearrow z^{*}$ for some $z^{*}$ with $z^{0}<z^{*}<e$ and $z^{*}=$ $\lim _{m \rightarrow \infty} z^{m+1}=\lim _{m \rightarrow \infty} T\left(z^{m}\right)=T\left(z^{*}\right)$.

(b) ClaIm. The solution $x(t, c)$ satisfies $0<z_{n}(t, c)<1$ for all $t \geqq 0$ and all $n \geqq 1$.

To verify this, we note that $\dot{z}_{n}=-\alpha_{n} z_{n}+f_{n}(x) \geqq-\alpha_{n} z_{n}$ so that $z_{n}(t) \geqq c_{n} \exp \left(-\alpha_{n} t\right)>0$. Let $y_{n}=1-z_{n}$ so that $\dot{y}_{n}=-\alpha_{n} y_{n}+g_{n}(y)$, where $g_{n}(y)=\alpha_{n}-\left(1+\exp \left(-\gamma_{n}-\sum_{m=1}^{\infty} \beta_{n m}+\sum_{m=1}^{\infty} \beta_{n m} y_{m}\right)\right)^{-1}$. Define $\widetilde{g}_{n}(y)=g_{n}(w)$, where $w_{n}=\max \left\{0, y_{n}\right\}$. Let $\dot{w}_{n}=-\alpha_{n} w_{n}+\widetilde{g}_{n}(w) \geqq$ $-\alpha_{n} w_{n}+\widetilde{g}_{n}(0)$. Thus $w_{n}(t) \geqq e^{-\alpha_{n} t}+\widetilde{g}_{n}(0) / \alpha_{n} \geqq \widetilde{g}_{n}(0) / \alpha_{n}>0$ for as long as $w_{n}(t)$ exists. This means that $\widetilde{g}_{n}(w(t))=g_{n}(w(t))$, so by uniqueness $0<w_{n}(t)=y_{n}(t)=1-z_{n}(t)$. This proves the claim.

(c) ClaIm. The critical point $x^{*}$ is asymptotically stable with respect to $\left\{x=\left(z_{1}, z_{2}, \cdots\right): 0<z_{n}<1\right\}$.

To prove this claim we define for stability of $x^{*}, u_{n}=z_{n}-z_{n}^{*}$ and $F_{n}(u)=f_{n}\left(u+x^{*}\right)-f_{n}\left(x^{*}\right)$ for $u=\left(u_{1}, u_{2}, u_{3}, \cdots\right)^{T}$. Then

$$
\left\{\begin{array}{l}
\dot{u}_{n}=-\alpha_{n} u_{n}+F_{n}(u) \\
u_{n}(0)=u_{n 0}=c_{n}-z_{n}^{*}
\end{array}\right.
$$

and $-1<-z_{n}^{*}<u_{n}(t)<1-z_{n}^{*}<1$ for all $t \geqq 0, n \geqq 1$. Let $v_{n}\left(u_{n}\right)=\left|u_{n}\right|$ and $v(u)=\left(v_{1}\left(u_{1}\right), v_{2}\left(u_{2}\right), \cdots\right)^{T}$. Then for some numbers $\delta_{n} \in[0,1)(d / d t) v_{n}\left(u_{n}(t)\right) \leqq$ $-\alpha_{n}\left|u_{n}\right|+\left|F_{n}(u)\right| \leqq-\alpha_{n} v_{n}+\sum_{m=1}^{\infty}\left(1+\exp \left(-\gamma_{n}-\sum_{j=1}^{\infty} \beta_{n j}\right)\right)^{-1} \delta_{n} \beta_{n m} v_{m}$. The Lyapunov function $v(t, x)=\sum_{n=1}^{\infty} \lambda_{n}\left|v_{n}\right|$ for the linear system $v_{n}^{\prime}=$ $-\alpha_{n} v_{n}+\sum_{m=1}^{\infty} \delta_{n} \alpha_{n} \beta_{n m} v_{m}$, and Theorem 6 imply that $u(t) \rightarrow 0$ as $t \rightarrow \infty$. 
REMARK 6. The analysis of this system in [4] proceeds under somewhat different assumptions. The assumptions $\beta_{n m} \geqq 0$, and $\sum_{m=1}^{\infty} \lambda_{m} \alpha_{m} \beta_{m n}<$ $\lambda_{n} \alpha_{n}$ are not used. It is assumed that $\sup \left|\gamma_{n}\right|=\gamma<\infty$, that $\sum_{m=1}^{\infty}\left|\beta_{n m}\right| \leqq 1$ for each $n \geqq 1$ and there is a $\delta$ such that $\alpha_{n}^{-1}(1+\exp (-1-\gamma))^{-1} \leqq \delta<1$ for all $n \geqq 1$. Under these conditions it is shown that there is a critical point $x^{*}$ which is stable (in the sense of the sup norm $\|x\|=\sup _{n}\left|z_{n}\right|$ ). Asymptotic stability is studied in [4] only for the finite approximations $\left(\Sigma_{N}\right)$.

5. Concluding remarks. Countably infinite systems of differential equations were viewed as an interconnection of countably infinite many free or isolated subsystems (described by ordinary differential equations defined on finite dimensional spaces). Well posedness results and several new stabilty results were established using scalar Lyapunov functions as well as vector Lyapunov functions. These were applied to several specific examples, including a neural model.

\section{REFERENCES}

[1] F. Brauer and J. A. Nohel, Qualitative Theory of Ordinary Differential Equations, W. A. Benjamin, Menlo Park, Calif., 1969 (see especially Chapter 5, Section 4).

[2] K. Deimling, Ordinary Differential Equations in Banach Spaces, Lecture Notes in Mathematics No. 596, Springer-Verlag, Berlin-Heidelberg-New York, 1977.

[3] M. Fiedler and V. Ptak, On matrices with non-positive off-diagonal elements and positive principal minors, Czechoslovak Math. J. 12 (1962), 382-400.

[4] K. V. Leung, D. Mangeron, M. N. Oguztörelli and R. B. Stein, On the stability and numerical solutions of two neural models, Utilitas Mathematica 5 (1974), 167-212.

[ 5] A. N. Michel ANd R. K. Mrller, Qualitative Analysis of Large Scale Dynamical Systems, Academic Press, New York, 1977.

[6] A. OSTRowSKI, Determinanten mit überwiegender Hauptdiagonale und die absolute Konvergenz von linearen Iterationsprozessen, Comment. Math. Helv. 30 (1956), 175-210.

[7] K. P. Persidskir, On stability of solutions of countable systems of differential equations, Izv. Akad. Nauk. Kazach SSR. 2 (1948), 3-35.

[8] K. P. Persidskir, Countable systems of differential equations and stability of their solutions III: Fundamental theorems on stability of solutions of countably many differential equations, Izv. Akad. Nauk Kazach SSR 9 (13), 1961, 11-34.

[9] A. P. Robertson and W. J. Robertson, Topological Vector Spaces, Cambridge University Press, No. 53, Cambridge, 1966.

[10] L. SHAw, Existence and approximation of solutions to an infinite set of linear timeinvariant differential equations, SIAM J. Appl. Math. 22 (2), March 1972, 266-279.

[11] L. SHAw, Solutions for infinite-matrix diffierential equations, J. Math. Anal. Appl. 41 (1973), 373-383.

[12] W. WALter, Differential and Integral Inequalities, Springer-Verlag, New York and Heidelberg, 1970.

[13] T. Yoshizawa, Asymptotic behavior of solutions of a system of differential equations, Contributions to Differential Equations 1 (1963), 371-387. 
R. K. MILLER AND A. N. MICHEL

Mathematics Department and Electrical Engineering Department Iowa State University AMES, Iowa 50011 U.S.A. AND ENGINEERING RESEARCH INSTITUTE Iowa State UnIVersity Ames, Iowa 50011 U.S.A. 